\title{
A importância de uma alimentação saudável como forma de aumento da imunidade
}

\section{através das vitaminas e minerais}

\author{
The importance of healthy eating as a way to increase immunity through vitamins and minerals
}

La importancia de una alimentación saludable como forma de aumentar la inmunidad a través de vitaminas y minerales

Recebido: 06/09/2021 | Revisado: 12/09/2021 | Aceito: 13/09/2021 | Publicado: 14/09/2021

Dandara Hillary de Souza Oliveira
ORCID: https://orcid.org/0000-0001-7200-7330
Centro Universitário Fametro, Brasil
E-mail: dandarathiago29@ gmail.com
Maria Izabele de Ouro da Silva
ORCID: https://orcid.org/0000-0003-4943-0290
Centro Universitário Fametro, Brasil
E-mail: mmariaizabele@ bol.com.br
Rita Gonzaga da Fonseca
ORCID: https://orcid.org/0000-0003-0867-6016
Centro Universitário Fametro, Brasil
E-mail: ritagonzaga007@ gmail.com
José Carlos de Sales Ferreira
CenciD: https://orcid.org/0000-0002-1867-8229
Centro Universitário Fametro, Brasil
E-mail: jcarlos.sales@ gmail.com

\begin{abstract}
Resumo
Introdução: Sabe-se que o sistema imune é constituído por uma complexa rede de células e moléculas responsáveis por identifica um antígeno e desencadear uma resposta efetora mediante a esse estímulo, as respostas são divididas, em dois sistemas, imunidade inata e imunidade adaptativa a inata apresenta uma resposta rápida para o organismo. Objetivo Geral: Identificar os benefícios da ingesta de vitaminas e minerais como B6, B9, C, D, e Zinco, através da alimentação. Metodologia: Para a realização desse estudo optou-se por uma pesquisa na modalidade de revisão integrativa de literatura. A revisão integrativa determina o conhecimento atual sobre uma temática específica, já que é conduzida de modo a identificar, analisar e sintetizar resultados de estudos independentes sobre o mesmo assunto. Resultados e Discussão: Todos os nutrientes, assim como as vitaminas e minerais são essenciais e cada um deles apresenta um papel fundamental para o organismo devendo receber maior atenção a suplementação desses nutrientes são essenciais para o bem-estar e o melhor funcionamento do organismo humano, contribuindo para a melhoria da qualidade de vida. Considerações finais: Através do desenvolvimento desse estudo se pode mostrar que através da alimentação pode-se alcançar maior qualidade de vida, já que é comprovado que com absorção das vitaminas e minerais o organismo humano tem melhor desenvolvimento, e por isso é muito importante que as pessoas possam adaptar-se a novos hábitos alimentares, a fim de evitar o surgimento de deficiências ou até mesmo patologias crônicas causadas pela falta de determinadas vitaminas e minerais.
\end{abstract}

Palavras-chave: Sistema imunológico; Nutrição; Nutrientes; Alimentação.

\begin{abstract}
Introduction: It is known that the immune system consists of a complex network of cells and molecules responsible for identifying an antigen and triggering an effector response through this stimulus, the responses are divided into two systems, innate immunity and adaptive to innate immunity presents a quick response to the body. General Objective: Identify the benefits of ingesting vitamins and minerals such as B6, B9, C, D, and Zinc, through food. Methodology: To carry out this study, we opted for a research in the modality of integrative literature review. The integrative review determines current knowledge on a specific topic, as it is conducted in order to identify, analyze and synthesize results of independent studies on the same subject. Results and Discussion: All nutrients, as well as vitamins and minerals are essential and each one of them plays a fundamental role for the body and should receive greater attention the supplementation of these nutrients are essential for the well-being and better functioning of the human body, contributing to the improvement of the quality of life. Final considerations: Through the development of this study, it can be shown that through nutrition, a better quality of life can be achieved, since it is proven that with the absorption of vitamins and minerals the human body has better development, and that is why it is very important that the people
\end{abstract}


can adapt to new eating habits in order to avoid the emergence of deficiencies or even chronic pathologies caused by the lack of certain vitamins and minerals.

Keywords: Immune system; Nutrition; Nutrients; Feeding.

\begin{abstract}
Resumen
Introducción: Se sabe que el sistema inmunológico consiste en una compleja red de células y moléculas encargadas de identificar un antígeno y desencadenar una respuesta efectora a través de este estímulo, las respuestas se dividen en dos sistemas, inmunidad innata y adaptativa a inmunidad innata presenta una rápida respuesta al cuerpo. Objetivo general: Identificar los beneficios de ingerir vitaminas y minerales como B6, B9, C, D y Zinc, a través de los alimentos. Metodología: Para la realización de este estudio se optó por una investigación en la modalidad de revisión integradora de la literatura. La revisión integradora determina el conocimiento actual sobre un tema específico, ya que se realiza con el fin de identificar, analizar y sintetizar resultados de estudios independientes sobre el mismo tema. Resultados y Discusión: Todos los nutrientes, así como las vitaminas y minerales son esenciales y cada uno de ellos juega un papel fundamental para el organismo y deben recibir una mayor atención la suplementación de estos nutrientes son fundamentales para el bienestar y mejor funcionamiento del ser humano. cuerpo, contribuyendo a la mejora de la calidad de vida. Consideraciones finales: A través del desarrollo de este estudio, se puede demostrar que a través de la nutrición se puede lograr una mejor calidad de vida, ya que está comprobado que con la absorción de vitaminas y minerales el cuerpo humano tiene un mejor desarrollo, y es por eso que Es muy importante que las personas se adapten a los nuevos hábitos alimentarios para evitar la aparición de deficiencias o incluso patologías crónicas provocadas por la falta de determinadas vitaminas y minerales.
\end{abstract}

Palabras clave: Sistema inmunológico; Nutrición; Nutrientes; Alimentación.

\title{
1. Introdução
}

As Vitaminas e Minerais determinam em grande parte a saúde e, influi no bem-estar e na capacidade cognitiva de uma pessoa, por isso uma boa alimentação pode ser o equilíbrio do corpo, carência de micronutrientes pode afetar todas as faixas etárias, e tendo assim muitos efeitos adversos na saúde dessas pessoas, dos quais nem todos são clinicamente aparentes. Mesmo com os níveis moderados de carência (que podem ser aprofundado por medições bioquímicas ou clínica) podem ter efeitos preocupantes na função humana (WHO \& FAO, 2006).

A vitamina B6, conhecida como piridoxina, é encontrada em abundancia em alimentos de origem animal e vegetal, portanto pode ser suprida ao organismo através da alimentação, ela participa da regulação de respostas imunológicas a várias doenças, A baixa concentração de vitamina B6 no organismo prejudica a proliferação dos linfócitos e reduz a produção de anticorpos (Priori et al., 2016).

A vitamina B9, ácido fólico ou folato, funciona como coenzima no metabolismo de aminoácidos e participa da síntese de purinas, pirimidinas e ácidos nucléicos DNA e RNA, além de ser vital para a divisão celular e a síntese proteica, ou seja, tem papel fundamental na multiplicação celular, no crescimento normal e na formação de anticorpos (Silva et al., 2015).

A vitamina $\mathrm{C}$ ou Ácido ascórbico é uma molécula neutra de cor branca, inodora e hidrossolúvel, produzida por plantas e muitos animais através de algumas passagens de biossíntese, essa vitamina é solvíveis em solventes orgânicos, com suas potentes propriedades antioxidante, e seu encargo no sistema imunológico tem aberto vários questionamentos e assim impulsionando vários estudiosos buscarem resposta sobre a função dessa vitamina para o fortalecimento do sistema imunitário e se é realmente fundamental para a homeostasia do organismo e se tem a capacidade de proteção contra agentes infecciosos e outras agressões do ambiente (Ribeiro, 2019).

A Vitamina D é uma vitamina lipossolúvel, adquirida a partir da reação, de uma radiação ultravioleta tipo B e ela acontece a partir de duas conformações, Ergocalciferol e a Colecalciferol são sintetizadas primariamente na pele. Essas duas vitaminas têm grande importância na função do organismo dos seres humanos como sua atuação na regulação e manutenção da quantidade de fósforo e cálcio, ressaltando que há poucos alimentos com esses nutrientes, por isso a suplementação se torna necessária em casos de deficiência dessa vitamina (Brito et al., 2017). 
Os mecanismos antioxidantes, nos quais o Zinco está envolvido, servem para estabilizar os radicais livres, mantendo a integridade funcional e estrutural das células e são extremamente importantes para o correto funcionamento do sistema imune (Borges \& Paschoal, 2013).

Sabe-se que o sistema imune é constituído por uma complexa rede de células e moléculas responsáveis por identifica um antígeno e desencadear uma resposta efetora mediante a esse estímulo, as respostas são divididas, em dois sistemas, imunidade inata e imunidade adaptativa a inata apresenta uma resposta rápida para o organismo. Em contraposição, a resposta imune adaptativa depende da ativação de células especializadas, os linfócitos, e das moléculas solúveis por eles produzidas (Cruvinel et al., 2010).

Todos os nutrientes, assim como as vitaminas e minerais são essenciais e cada um deles apresenta um papel fundamental para o organismo devendo receber maior atenção a suplementação desses nutrientes são essenciais para o bemestar e o melhor funcionamento do organismo humano, contribuindo para a melhoria da qualidade de vida. O projeto visa atribuir conhecimento mútuo, alcançando a sociedade como um todo, com o intuito principal de identificar agravos na saúde do sistema imune, destacando os benefícios para o aumento e manutenção da imunidade com o emprego da suplementação e adequação da alimentação no cotidiano.

Nesse sentido, o objetivo geral deste artigo é identificar os benefícios da ingesta de vitaminas e minerais como B6, B9, C, D, e Zinco, através da alimentação.

\section{Metodologia}

Para a realização desse estudo optou-se por uma pesquisa na modalidade de revisão integrativa de literatura. A revisão integrativa determina o conhecimento atual sobre uma temática específica, já que é conduzida de modo a identificar, analisar e sintetizar resultados de estudos independentes sobre o mesmo assunto (Silveira et al., 2008).

A Revisão Integrativa é um método de pesquisa apontado como ferramenta de grande relevância no campo da saúde, por proporcionar a busca, a avaliação crítica e a síntese de evidências sobre um tema investigado. Esses aspectos facilitam a identificação dos resultados relevantes, de lacunas que direcionam para o desenvolvimento de futuras pesquisas e auxiliam o profissional a escolher condutas e a tomar decisões, proporcionando um saber crítico (Souza, Silva \& Carvalho, 2010).

Para extrair os dados dos artigos selecionados, foi necessário a utilização de um instrumento previamente elaborado capaz de assegurar que a totalidade dos dados relevantes seja extraída, minimizar o risco de erros na transcrição, garantir precisão na checagem das informações e servir como registro (Souza, Silva \& Carvalho, 2010).

A coleta das informações para a pesquisa bibliográfica se deu por meio da exploração da base de dados da Biblioteca Virtual em Saúde (BVS), Biblioteca Cientifica Eletrônica Virtual (SCIELO) e Literatura Latino-Americana e do Caribe em Ciências da Saúde (LILACS).

A busca na base de dados foi orientada pelos descritores: vitaminas, alimentação saudável, sistema imunológico, e será realizado em todos os índices, buscando captar o maior número de artigos publicados no período proposto que abordem a temática em discussão, visando o desenvolvimento fidedigno desse estudo.

Para uma análise crítica e reflexiva dos estudos incluídos na revisão, foi realizada uma leitura minuciosa e criteriosa destacando os que atingirem os critérios de inclusão e que contemplarem o objetivo proposto, para viabilizar o resultado da pesquisa de forma objetiva.

Foram incluídos os artigos publicados em língua portuguesa nos últimos cinco anos, com texto completo, disponível on-line, com acesso livre. Foram excluídos da amostra os artigos publicados em línguas estrangeiras, os que não apresentarem o texto na integra, artigos que não apresentavam relação direta com o tema, resumos, monografias, dissertações, teses e artigos repetidos. 


\section{Resultados e Discussão}

\subsection{Vitamina B6 e seus benefícios}

Segundo alguns estudiosos a vitamina B6 (piridoxina) pode ser um potencial candidato para o tratamento de algumas doenças como, úlceras diabetes e Aterosclerose na Diabetes (KELSO et al., 2016). E além de produzir energia, é importante para formação dos glóbulos vermelhos e ajuda o sistema imunológico a partir da produção de anticorpos (ROY, 2017). Hábitos saudáveis como realizar atividades físicas regularmente, alimentação balanceada em nutrientes, boa qualidade de sono, bom relacionamento com familiares e comunidade próxima, são vistos como fortalecedores do sistema imunológico e, sendo assim, auxiliam na prevenção de doenças (Kim \& Su, 2020).

A forma metabolicamente ativa da vitamina B6 é o piridoxal fosfato (PLP), que funciona como uma co-enzima de várias enzimas envolvidas em praticamente todas as reações no metabolismo dos aminoácidos, assim como em vários aspectos do metabolismo de neurotransmissores, glicogênio, esfingolipídios, heme e esteróides; desta forma, a vitamina B6 é essencial para uma variedade de transaminases, descarboxilase, racemases e isomerases de aminoácidos. É necessária para a biossíntese de neurotransmissores serotonina, epinefrina, norepinefrina e ácido gama-aminobutírico, o vasodilatador e secretagogo gástrica histamina e os precursores porfirina de heme (Fitzpatrick, 2017).

A vitamina B6 é necessária para o funcionamento adequado de mais de sessenta enzimas e essencial para a síntese normal do ácido nucléico e das proteínas. Participa da multiplicação de todas as células e da produção das hemácias e das células do sistema imunológico. Influencia o sistema nervoso através de seus efeitos sobre vários minerais e neurotransmissores cerebrais. A piridoxina é encontrada em maior proporção em alimentos de origem animal (carnes, de porco principalmente), leite e ovos. Entre os vegetais, assinala-se a batata inglesa, aveia, banana e gérmende trigo (Hendler, 2016).

\subsection{A vitamina B9 e suas funções no sistema imune}

O ácido fólico (vitamina B9), é necessário para inúmeras funções do corpo, suas ações neuroprotetoras prevenindo os danos causados por hiper-homocisteinemia aguda in vivo, (KRONENBERG et al., 2009), essa vitamina é um nutriente essencial na dieta humana envolvida em inúmeras reações metabólicas com a síntese do DNA e em diversos crescimento celular e sem contar nas novas produção de proteína, e formação de emacias (ZHAO et al., 2016).

O fato de os glóbulos vermelhos ficarem maiores e menos resistentes, é pela carência de vitamina B9, que é a responsável pela produção e maturação de células saudáveis. Se essa vitamina está em falta, a síntese de ácido desoxirribonucléico (DNA), que armazena informações genéticas, acaba sendo prejudicada, e os mecanismos genéticos de maturação e divisão nuclear, não funcionam normalmente (ZAGO et al., 2017).

As melhores fontes de B9 ou ácido fólico como é conhecido, são fígado, rim, feijão, vegetais de cor escura sobretudo espinafres, espargos e brócolis, carne de vaca magra, batatas, pão de trigo completo e feijões secos são boas fontes de ácido fólico (CARMO, 2017).

\subsection{Vitamina C e seu potente antioxidante}

A função da vitamina C, no Sistema imunitário é fundamental para a homeostasia do organismo e tem a capacidade de conferir proteção contra agentes infecciosos e outras agressões, o aumento da concentração de glóbulos brancos desempenha importantes funções na linha de defesa do corpo, tal como o apoio dos antioxidantes para a proteção do sistema imunológico, reduzindo assim, a morte celular (Medina, 2019).

O aumento da concentração de vitamina C, desempenha um papel importante nessas células, como proteção antioxidante durante a fagocitose, migração e redução da intensidade da cascata inflamatória, pelo controle do balanço das indicações pró-inflamatórias e anti-inflamatórias, como pode ser observado no estudo de (Theilla et al., 2016). 
A vitamina $\mathrm{C}$ é encontrada principalmente em frutas e hortaliças. Esta é encontrada em grande concentração nas células imunes, porém na presença de infecções e distúrbios que aumentam o nível de estresse, essa concentração pode diminuir rapidamente. Possui atividade antioxidante, a qual evita o dano oxidativo das células imunes. Ela também auxilia nas funções dos fagócitos, a produção de citocinas, a proliferação de linfócitos T e a expressão gênica das moléculas de adesão dos monócitos (Sorice, 2017).

A vitamina $\mathrm{C}$ é de extrema importância para o desenvolvimento humano, pois ela é responsável pelo nosso sistema imune e pode ser facilmente encontrada em frutas cítricas e ela desempenha um importante papel no nosso desenvolvimento (Domingues, 2016).

\subsection{Vitamina $D$ e seus benefícios no organismo}

As pesquisas atuais indicam que a vitamina $\mathrm{D}$ e seus análogos não só previnem o desenvolvimento de doenças autoimunes como também poderiam ser utilizados no seu tratamento (Amado et al., 2016). A vitamina D é um nutriente que além das funções exemplares de homeostase do cálcio e fósforo e do metabolismo ósseo, também tem enorme impacto na resposta imunológica (Gonçalves-Mendes et al., 2019).

Concentrações de vitamina D mostram efeitos protetores para a redução de eventos cardiovasculares, de câncer colorretal e de todas as causas de mortalidades, foi feita uma revisão sistemática e experimentos randomizados sobre o consumo da vitamina $\mathrm{D}$ e foram comprovados os seus benefícios na força muscular, no equilíbrio e na prevenção de quedas e fraturas (Galvão, et al., 2016).

A vitamina D é essencial para o funcionamento cognitivo, pois há receptores no cérebro que tem o papel de processar e de desenvolver novas memórias, sendo de extrema importância para prevenção de demências. Confirmando estas informações estudos apontam que a concentração de vitamina D em indivíduos com Alzheimer é baixa, situação que pode ocorrer devido à baixa ingestão desta vitamina ou baixa exposição ao sol (Correia, 2016).

\subsection{O zinco e sua importância para a saúde do corpo}

De acordo com Barnett (2016), o zinco é um nutriente importante do metabolismo humano, nos quais analisou o aumento da função das células $\mathrm{T}$ em idosos com o consumo de zinco através de alimentos ricos nesse nutriente, e aumento na porcentagem de linfócitos, estando o zinco interligado a uma formula à base de óleo de peixe, nessa ordem.

Estudos assinalam que o zinco participa da constituição de várias enzimas, muitas delas envolvidas no mecanismo de carboidratos, proteínas e lipídios e na síntese e degradação de ácidos nucléicos (Fukada et al., 2017). Em aproximadamente $70 \%$ destas enzimas, o zinco tem uma função de catalisador, mas também pode ter papel estrutural, agir como substrato ou atuar como regulador da atividade de enzimas (Andreini \& Bertni, 2016).

Dentre as principais funções do zinco, o destaque maior é na participação na síntese e degradação de carboidratos, lipídios e proteínas, na manutenção e crescimento do desenvolvimento normal no funcionamento adequado do sistema imunológico na defesa antioxidante, função neurossensorial e também na transcrição e tradução de polinucleotidios (Triebsees, 2016).

\section{Considerações Finais}

Através do desenvolvimento desse estudo se pode mostrar que através da alimentação pode-se alcançar maior qualidade de vida, já que é comprovado que com absorção das vitaminas e minerais o organismo humano tem melhor desenvolvimento, e por isso é muito importante que as pessoas possam adaptar-se a novos hábitos alimentares, a fim de evitar o surgimento de deficiências ou até mesmo patologias crônicas causadas pela falta de determinadas vitaminas e minerais. 
Pode-se ainda, contribuir para o conhecimento em geral sobre os benefícios de uma alimentação saudável, assim como déficit causado pela alimentação inadequada, relacionar a boa alimentação com o bom funcionamento do metabolismo humano e sistema imunitário, enfatizando que vitaminas e minerais têm papel fundamental na manutenção do sistema imune.

\section{Referências}

Abbas, A. K., Lichtman, A. H., \& Pillai, S. (2015). Imunologia Celular e Molecular. (8a ed.), Elsevier.

Abrahamsohn. (2016). Histologia. Guanabara Koogan,

Arosa, F. A., \& Cardoso E. M. P. F. (2012). Fundamentos de Imunologia. edições técnicas L, editor. Lidel.

Andreni, C., \& Bertini, I. (2016). A bioinformaticsviewofzincenzymes. J. Inorg. Biochem, 111, 150-156.

Aleluia, M. A. D. C., Mendes, J. P. C., \& Silva, A. Q. G. (2016). Efeitos da suplementação de glutamina sobre o sistema imune em atletas submetidos ao treinamento excessivo: uma revisão sistemática. Revista Brasileira de Nutrição Esportiva, 10(60).

Biasebetti, M. D. B. C., Rodrigues, I. D., \& Mazur, C. E. (2018). Relação do consumo de vitaminas e minerais com o sistema imunitário: uma breve revisão. Visão Acadêmica, 19(1).

Beard, C. et al. (2016). Isexcessfolicacidsuplementation a riskfactor for autism? Med Hypotheses, 77(1), PP, 15-17

Borges, L. E. M., \& Paschoal, J. J. (2013). Influência dos micro-minerais (Cu, Mn, Se e Zn) no sistema imunológico dos bovinos. Cadernos de Pós-Graduação da FAZU, 3.

Bhasin, A., \& Rao, M. Y. (2013). Características da anemia em idoso: estudo de base hospitalar no sul da Índia.

Brodin, P., Jojic, V., Gao, T., et al. (2015). Variation in theHumanImmune System IsLargelyDrivenby Non-HeritableInfluences. The Cell, 160(.1-2), 37-47.

Coico, R., et al. (2015). Imunolgia. Tradução Eiler Fritsch Toros. Guanabara Koogan.

Cordeiro, C. A. et al. (2010). Imunologia da retinocoroiditetoxoplásmica. Arquivo Brasileiro de Oftalmologia. 73(6), 548- 551.

Correia J., et al. (2016). Programa nacional para promoção da alimentação saudável nutrição e doença de Alzheimer. Nutrição Ativas. 17(2), 455-468.

Costa, C. L., \& Fonseca, F. C. P. (2017). Influência da nutrição sobre o sistema imune intestinal. Ceres: nutrição \& saúde, 5(3).

Carmo, I. (2017). Equilibriu vital: vitaminas e minerais. Publicações. Dom Quixote. Portugal.

Cruvinel, W. M. et al. (2010). Sistema Imunitário - Parte I Fundamentos da imunidade inata com ênfase nos mecanismos moleculares e celulares da resposta inflamatória. Revista Brasileira de Reumatologia, 50(4), 61 - 434.

Domingues, G. (2017). Relação entre medicamentos e ganho de peso em indivíduos portadores de autismo e outras síndromes relacionadas. Nutrição Ativas. $17(2), 455-468$

Fernandes, T. Fe. (2017). Impactos da microbiota intestinal na saúde do lactente e da criança em curto e longo prazo. International Journal of Nutrology, $10(\mathrm{~S} 01)$.

Fonseca, F. C. P. D. (2016). Influência da nutrição sobre o sistema imune intestinal. CERES: Nutrição \& Saúde, 5(3).

Fitzpatric, T. B., Basset G. J. C., Borel P., Carrari F., Della Penna D., Fraser, P. D., Hellmann, H., Osorio, S., Rothan C., Valpuesta V., Caris-Veyrat, C., \& Fernie, A. R. (2017). VitaminDeficiencies in Humans: Can Plant Science Help? February, The Plant Cell, $24,395-414$.

Fukada, T., Nishida, K., Murakami, M., \& Hirano, T. (2017). Zinc homeostasisandsignalig in heathanddiseases- zincsignaling. Journal of Biological Inorganic Chemistry, 16, 1123- 1134 .

Gredel, S. (2012). Nutrição e imunidade no homem. 2. ed. Bélgica: IlsiEuropeConciseMonograph Series, 32.

Gouba, N., et al. (2019). Digestive tract mycobiota and microbiota and the effects on the immune system. Human Microbiome Journal, 12.

Hall, John E. (2011). 1946 - Tratado de Fisiologia Médica (12a ed.), Elsevier.

Hendler S. S. (2016). Vitaminas e saúde. Jornal de pediatria. Rio de Janeiro. 1(2), 34-36.

Kelso, B et al. Pyridoxinerestoresendothelialcellfunction in high glucose MetabSyndrDisord, 9 (1), 63-68, 2016.

Kim, S. W., \& Su, K. P. (2020). Usingpsychoneuroimmunityagainst COVID-19. Brain, Behavior, andImmunitty, (pré-publicação).

Kronenberg, G., Colla, M., \& Endres, M. (2009). Folicacid neuro degenerative and neuropsychiatric disease. Curr Mol Med, 9(3), 315-23.

Lemos, A. (2010). Processos naturais de desintoxicação - para prevenção e tratamento de doenças.

Mahan, L., et al. (2018). Krause alimentos, nutrição e dietoterapia. [tradução Verônica Mannarino, Andrea Favano] (8a ed.), Elsevier. 
Mariano, K. P., \& Fioravanti, M. C. S. (2016). Nutrição e função imune. Universidade Federal de Goiás, Escola de Veterinária e Zootecnia. Programa de pósgraduação em ciência animal.

Mazur, C. E, Rodrigues, I. D, \& Biasebetti, M. B. S. (2018). Relação do consumo de vitaminas e minerais com o sistema imunitário: uma breve revisão. Visão Acadêmica, 19(1).

Molina, N., Morandi A. C., Bolin A. P., \& Otton, R. (2014). Comparativeeffectoffucoxanthinandvitamin C onoxidativeandfunctionalparametersofhumanlymp hocytes. IntImmunopharmacol.

Parham, Peter. (2011). O Sistema imune. [tradução: Christian Viezzer...et al.] ; revisão técnica: Denise Cantarelli Machado. (3a ed.), Artmed xx, 588.

Pogliarone, A.C. (2012). Imunidade Inata. Mestrado. 6f. Programa de pós-graduação em imunologia básica aplicada.

Perbelin, D. S., Angélica, et al. (2019). The role of microbiota as allied in the immune system. Arquivos do MUDI, 23(3).

Roy. V. (2017). Food andNutritionCommubication. Nestlé Suisse S.A.

Sarni, R. O., Souza, F. I., Cocco, R. R., Mallozi, M. C., \& Solé, D. (2016). Micronutrientes e sistema imunológico. Rev Bras Alerg Imunopatol, 33(1).

Santos, L. M. P., \& (2011). Pereira, M. Z. Efeito da fortificação com ácido fólico na redução dos defeitos neural. CAD. Saúde Pública. Rio de Janeiro, 23(1).

Silva, S. M. C., et al. (2010). Seabra da Tratado de Alimentação, nutrição e dietoterapia. (2a ed.), Roca.

Sorice, A., Guerriero E., Capone F., et al. (2016). Ascorbicacid: its role in imune systemnd chronicin flammation diseases. Mini-Reviews in Medicinal Chemistry, 14(5), 444-52.

Faustino, T. N. (2012). Nutrição Clínica. Guanaraba Koogan.

Theilla, Miriam et al. (2016). "Ácidos graxos n-3 enterais e micronutrientes aumentam a porcentagem de neutrófilos positivos e moléculas de adesão de linfócitos: um mediador potencial de cicatrização de úlceras de pressão em pacientes criticamente enfermos. The British Journal of Nutrition, 107(7).

Uehara, S. K., \& Rosa, G. (2010). Associação da deficiência de ácido fólico com alterações patológicas e estratégias para sua prevenção: uma visão crítica. Rev. Nutr., Campinas, 23(5).

Vannucchi, H., \& Rocha, M. de M. (2012). Vitamina C (ácido ascórbico). ILSI Brasil - International Life Sciences Institute from Brasil. 2, 1-12.

Vidigal, F. C. (2010). Deficiência de Vitamina B12 e ácido Fólico e sua relação com a aterosclerose. Minas Gerais: GANEP- Grupo de Apoio a Nutrição Enteral e Parenteral.

Wilson, M. P., Mills. P. B., \& Clayton, P. T. DIsordensAffecting Vitamina B6 metabolismo JOURNAL Ofinheritedmetabolicdiseaseb, 1-18-23019.

Wu, D.; LEWIS, E. D.; Pae, M. et al. (2019). Nutritional Modulation of Immune Function: AnalysisofEvidence, Mechanisms, and Clinical Relevance. Frontiers in Immunology, 9, artigo 3160.

Zhao, Y., Huang, G., Chen, S., Gou, Y., Dong, Z., \& Zhang, X. (2016). Folicaciddeficiencyincreasesbraincellinjury via autophagyenhancementafter focal cerebral ischemia. J NutrBiochem. 38, 41-9.

Zago, M. A.; Falcâo, R. P.; \& Pasquini, R. (2017). Carência de folatos (B9). Anemias Megaloblásticas. Tratado de hematologia, cap 18. 